\title{
Abundant M-fractional optical solitons to the pertubed Gerdjikov-Ivanov equation treating the mathematical nonlinear optics
}

\author{
Asim Zafar ${ }^{1}$, Khalid K. Ali ${ }^{2}$, M. Raheel ${ }^{1}$, Kottakkaran Sooppy Nisar ${ }^{3}$ and Ahmet Bekir ${ }^{4, *}$ \\ ${ }^{1}$ Department of Mathematics, COMSATS University, Vehari Campus, Pakistan. \\ ${ }^{2}$ Department of Mathematics, Faculty of Science, Al-Azhar University, \\ Nasr-City, Cairo, Egypt. \\ ${ }^{3}$ Department of Mathematics, College of Arts and Sciences, \\ Wadi Aldawaser 11991, Prince Sattam bin Abdulaziz University, Saudi Arabia. \\ ${ }^{4}$ Neighbourhood of Akcaglan, Imarli Street, Number: 28/4, 26030, Eskisehir, Turkey.
}

\begin{abstract}
In this paper, the perturbed Gerdjikov-Ivanov (GI) equation using a truncated Mfractional derivative is studied in mathematical nonlinear optics. We explore its novel dark and other soliton solutions and compared them with the existing results. To obtain the objective, two particular methods, modified extended tanh expansion method and $\operatorname{Exp}_{a}$ function method, are implemented. In this exert, a arrangement of exact solitons are received as well as verified by utilizing the MATHEMATICA software. The dynamical characteristics of the obtained results, along with a fractional parameter, are also discussed via two and three-dimensional graphs. These solutions suggest that the employed methods are impressive, determined and smooth as compared to many other methods. The work of this paper is of high importance regarding its applications in photonic crystal fibers and mathematical physics.
\end{abstract}

Keywords: M-fractional derivative; perturbed Gerdjikov-Ivanov Equation; Optical solitons.

\section{Introduction}

Many phenomenon are often described in the form of nonlinear Schrödinger (NLS) equations. Then those NLS equations are studied for different purposes with different approach. These have much importance in the different fields of science and engineering. In recent years, to solve the different NLS equations different analytical and numerical approaches

\footnotetext{
*Corresponding author: bekirahmet@gmail.com
} 
have been developed [22-25]. The NLS equations that are mostly studied are those have cubic nonlinearity. For example, NLS equation along quintic non-linearity is the perturbed GI equation.

Various approaches have been applied to find different exact solitons of this equation. For Instance, two types of bright wave solutions of perturbed GI equation have been obtained with the help of semi-inverse variational method [16]. Distinct solitons are investigated by applying the sine-Gordon equation method [17]. Biswas et al. have obtained the singular and bright solitons with the use of extended trial equation approach for the perturbed GI equation [18]. Different solitary wave solutions of the perturbed GI equation have been found by the implementation of the $\exp (\phi(\xi))$-expansion and the Kudryashov techniques [19]. Various wave solutions have obtained by applying the two different method, $\exp (\phi(\xi))$-expansion method and $\left(G^{\prime} / G^{2}\right)$-expansion technique [20]. Different optical solitons have been determined by using the $\exp _{a}$ function method and the modified Kudryashov method [21]. Recently, some novel wave solutions were investigated by using the generalized exponential rational function method [26].

Besides of these methods, there are two methods that are more reliable, simple and useful named as: modified extended tanh expansion method (EThEM) and Exp function method. The modified extended tanh expansion technique [1] has been used to discuss Biswas and Arshed model with non-linearity factor " $n$ ". The different solitons of $(2+1)$ dimensional integrable nonlinear Schrödinger equation were explained in [2]. Various solitons of new coupled evolution equation were explained [4]. Optical soliton solutions of the travelling wave nonlinear equations have been determined in [5-8]. Explicit exact solitons of two nonlinear Schrödinger equations have investigated through two different techniques [13]. Similarly, this techniques have been applied to solve the other many NLS equations $[9,11]$.

The main task in this research is to search some new dark and other optical soliton solutions of the perturbed GI equation with truncated M. fractional derivative. The modified extended tanh expansion method and $E x p_{a}$ function method are employed to acquire the aforesaid task.

\section{The governing model and the mathematical analysis}

The perturbed Gerdjikov-Ivanov (GI) equation can be read as [15-21]:

$$
\iota g_{t}+\tau_{1} g_{x x}+\tau_{2}|g|^{4} g-\iota\left(\tau_{3} g^{2} g_{x}^{*}+\theta_{1} g_{x}+\theta_{2}\left(|g|^{2} g\right)_{x}+\rho|g|_{x}^{2} g\right)=0
$$

here $g=g(x, t)$ shows the complex-valued wave function, depends on independent variables $x$ and $t$. In Eq. (1), $\tau_{1}$ shows the coefficient of GVD, $\tau_{2}$ represents the coefficient of the quintic non-linearity of the model and $\tau_{3}$ indicates the coefficient of the nonlinear dispersion term. Moreover, the parameters $\theta_{1}, \theta_{2}$ and $\rho$ represent the perturbation effects. Finally, the term $g^{*}$ shows the complex conjugate of $g$. Eq. (1) with truncated M-fractional derivative 
is given as:

$$
\begin{aligned}
i D_{M, t}^{\mu, \beta} g+\tau_{1} D_{M, x}^{2 \mu, \beta} g+\tau_{2}|g|^{4} g & =i\left(\tau_{3} g^{2} D_{M, x}^{\mu, \beta} g^{*}+\theta_{1} D_{M, x}^{\mu, \beta} g+\theta_{2} D_{M, x}^{\mu, \beta}\left(|g|^{2} g\right)\right. \\
& \left.+\rho g D_{M, x}^{\mu, \beta}|g|^{2}\right), \quad \mu \in(0,1), \quad \beta>0
\end{aligned}
$$

where

$$
D_{M, t}^{\mu, \beta} g=\lim _{\tau \rightarrow 0} \frac{g\left(x, t E_{\beta}\left(\tau t^{1-\mu}\right)\right)-g(x, t)}{\tau}, \quad \mu \in(0,1), \quad \beta>0,
$$

that $E_{\beta}($.$) is a truncated Mittag-Leffler function of one parameter [14].$

Now by using the complex constraints conditions given in the following:

$$
\begin{aligned}
& g(x, t)=G(\zeta) e^{i \psi}, \quad \zeta=\frac{\Gamma(\beta+1)}{\mu}\left(x^{\mu}-\lambda t^{\mu}\right), \\
& \psi=\frac{\Gamma(\beta+1)}{\mu}\left(-\sigma_{1} x^{\mu}+\sigma_{2} t^{\mu}\right),
\end{aligned}
$$

Here $\lambda$ indicates the phase component, $\sigma_{1}$ shows the frequency of the wave solutions and $\sigma_{2}$ represents the wave number of solitons. By putting the Eq. (4) into the model Eq. (2), we get:

Real part:

$$
\tau_{1} G^{\prime \prime}-\left(\sigma_{2}+\tau_{1} \sigma_{1}^{2}+\theta_{1} \sigma_{1}\right) G+\left(\tau_{3}-\theta_{2}\right) \sigma_{1} G^{3}+\tau_{2} G^{5}=0
$$

Imaginary part:

$$
\left(\lambda+\theta_{1}+2 \tau_{1} \sigma_{1}\right)+\left(\tau_{3}+3 \theta_{2}+2 \rho\right) G^{2}=0
$$

From Eq.(6), we get:

$$
\lambda=-\left(\theta_{1}+2 \tau_{1} \sigma_{1}\right) ; \quad \rho=-\frac{1}{2}\left(\tau_{3}+3 \theta_{2}\right)
$$

Using the terms $G^{\prime \prime}$ and $G^{5}$ and the homogenous balance approach, we observe $m=1 / 2$. Therefore, we use the, we use the following transformation to get solution in retrieve form:

$$
Q(\zeta)=G^{\frac{1}{2}}(\zeta)
$$

By using the Eq. (8) into Eq. (5), yields

$$
\tau_{1}\left(2 Q Q^{\prime \prime}-\left(Q^{\prime}\right)^{2}\right)-4\left(\sigma_{2}+\tau_{1} \sigma_{1}^{2}+\theta_{1} \sigma_{1}\right) Q^{2}+4\left(\tau_{3}-\theta_{2}\right) \sigma_{1} Q^{3}+4 \tau_{2} Q^{4}=0
$$

\section{Application of the modified EThEM:}

Here, a quick review of the said method and its implementation both are explained. Let's assume the below non-linear PDE of the form:

$$
Y\left(u, u^{2} u t, u_{x}, u_{t t}, u_{x x}, u_{x t}, \ldots\right)=0
$$


here $u$. Let us assume the below wave transformation:

$$
u(x, t)=U(\zeta), \quad \zeta=x-\nu t
$$

Here $\nu$ shows the wave velocity. Putting the Eq. (11) into Eq. (10), taking the following nonlinear ODE:

$$
\chi\left(U, U^{2} U^{\prime}, U^{\prime \prime}, \ldots\right)=0 .
$$

Here primes represents the derivatives w.r.t $\zeta$.

Moreover, consider the solution of Eq. (12) is of the form:

$$
U(\zeta)=\alpha_{0}+\sum_{n=1}^{m} \alpha_{n} \phi^{n}(\zeta)+\sum_{n=1}^{m} \beta_{n} \phi^{-n}(\zeta)
$$

In Eq. (13), $\alpha_{0}, \alpha_{n}, \beta_{n},(n=1,2,3, \ldots, m)$ are undetermined and to be find later. Notice that $\alpha_{n}$ and $\beta_{n}$ are not both zero at a time. By balancing nonlinear term and the highest derivative in Eq. (12), we get $m$.

The function $\phi(\xi)$ satisfied:

$$
\phi^{\prime}(\zeta)=\Omega+\phi^{2}(\zeta)
$$

with $\Omega$ as a unknown parameter and the Eq. (14) have the below form of solutions [3]:

(i) if $\Omega<0$, we get

$$
\phi(\zeta)=-\sqrt{-\Omega} \tanh (\sqrt{-\Omega} \zeta)
$$

or

$$
\phi(\zeta)=-\sqrt{-\Omega} \operatorname{coth}(\sqrt{-\Omega} \zeta)
$$

(ii) if $\Omega=0$, we get

$$
\phi(\zeta)=\frac{-1}{\zeta}
$$

(iii) if $\Omega>0$, we get

$$
\phi(\zeta)=\sqrt{\Omega} \tan (\sqrt{\Omega} \zeta)
$$

or

$$
\phi(\zeta)=-\sqrt{\Omega} \cot (\sqrt{\Omega} \zeta) .
$$

Putting Eq. (13) and it's derivatives into Eq. (12) along Eq. (14), yields polynomials in powers of $\phi(\zeta)$. Sorting the coefficients of each power of $\phi(\zeta)$ and letting each summation equal to 0 , we get a set of algebraic equations for $\alpha_{0}, \alpha_{n}, \beta_{n}(n=1,2,3, \ldots, m)$, and $\Omega$. Writing the values of these parameter into Eq. (13) with value of $m$, yields the solutions to Eq. (10).

Now by using the homogenous balance technique on Eq. (9), we achieve $m=1$. Then, Eq. (13) becomes:

$$
Q(\zeta)=\alpha_{0}+\alpha_{1} \phi(\zeta)+\frac{\beta_{1}}{\phi(\zeta)}
$$


Here $\alpha_{0}, \alpha_{1}$ and $\beta_{1}$ are undermined parameters. By inserting the Eq. (20) and Eq. (14) into Eq. (9), sorting the coefficients of each power of $\phi(\zeta)$, we obtain the set of equations having $\alpha_{0}, \alpha_{1}, \beta_{1}$ and other parameters. By using the soft computation, we gain the following sets:

Set 1:

$$
\begin{aligned}
\left\{\alpha_{0}\right. & =\frac{3 \sigma_{1}\left(\theta_{2}-\tau_{3}\right)}{8 \tau_{2}}, \alpha_{1}=\mp \frac{i \sqrt{3} \sqrt{\tau_{1}}}{2 \sqrt{\tau_{2}}}, \beta_{1}=0 \\
\sigma_{2} & \left.=-\frac{\sigma_{1}\left(-6 \theta_{2} \sigma_{1} \tau_{3}+3 \theta_{2}^{2} \sigma_{1}+16 \theta_{1} \tau_{2}+\sigma_{1}\left(3 \tau_{3}^{2}+16 \tau_{1} \tau_{2}\right)\right)}{16 \tau_{2}}, \Omega=\frac{3 \sigma_{1}^{2}\left(\theta_{2}-\tau_{3}\right)^{2}}{16 \tau_{1} \tau_{2}}\right\}
\end{aligned}
$$

We now using the Eq. (21) and Eqs. (15)-(19) in Eq. (20), and argue the following cases. if $\Omega<0$, then

$$
\begin{aligned}
g_{1}(x, t) & =\left\{\frac{3 \sigma_{1}\left(\theta_{2}-\tau_{3}\right) \pm 4 i \sqrt{3} \sqrt{\tau_{1}} \sqrt{\tau_{2}} \sqrt{-\Omega} \tanh (\zeta \sqrt{-\Omega})}{8 \tau_{2}}\right\}^{\frac{1}{2}} \\
& \times \exp \left(\iota \frac{\Gamma(\beta+1)}{\mu}\left(-\sigma_{1} x^{\mu}+\sigma_{2} t^{\mu}\right)\right)
\end{aligned}
$$

or

$$
\begin{aligned}
g_{2}(x, t) & =\left\{\frac{3 \sigma_{1}\left(\theta_{2}-\tau_{3}\right) \pm 4 i \sqrt{3} \sqrt{\tau_{1}} \sqrt{\tau_{2}} \sqrt{-\Omega} \operatorname{coth}(\zeta \sqrt{-\Omega})}{8 \tau_{2}}\right\}^{\frac{1}{2}} \\
& \times \exp \left(\iota \frac{\Gamma(\beta+1)}{\mu}\left(-\sigma_{1} x^{\mu}+\sigma_{2} t^{\mu}\right)\right) .
\end{aligned}
$$

If $\Omega>0$, then

$$
\begin{aligned}
g_{3}(x, t) & =\left\{\frac{3 \sigma_{1}\left(\theta_{2}-\tau_{3}\right) \mp 4 i \sqrt{3} \sqrt{\tau_{1}} \sqrt{\tau_{2}} \sqrt{\Omega} \tan (\zeta \sqrt{\Omega})}{8 \tau_{2}}\right\}^{\frac{1}{2}} \\
& \times \exp \left(\iota \frac{\Gamma(\beta+1)}{\mu}\left(-\sigma_{1} x^{\mu}+\sigma_{2} t^{\mu}\right)\right),
\end{aligned}
$$

or

$$
\begin{aligned}
g_{4}(x, t) & =\left\{\frac{3 \sigma_{1}\left(\theta_{2}-\tau_{3}\right) \pm 4 i \sqrt{3} \sqrt{\tau_{1}} \sqrt{\tau_{2}} \sqrt{\Omega} \cot (\zeta \sqrt{\Omega})}{8 \tau_{2}}\right\}^{\frac{1}{2}} \\
& \times \exp \left(\iota \frac{\Gamma(\beta+1)}{\mu}\left(-\sigma_{1} x^{\mu}+\sigma_{2} t^{\mu}\right)\right) .
\end{aligned}
$$

Set 2:

$$
\begin{gathered}
\left\{\alpha_{0}=\frac{3 \sigma_{1}\left(\theta_{2}-\tau_{3}\right)}{8 \tau_{2}}, \alpha_{1}=\mp \frac{i \sqrt{3} \sqrt{\tau_{1}}}{2 \sqrt{\tau_{2}}}, \beta_{1}= \pm \frac{3 i \sqrt{3} \sigma_{1}^{2}\left(\theta_{2}-\tau_{3}\right)^{2}}{128 \sqrt{\tau_{1}} \tau_{2}^{3 / 2}}, \Omega=-\frac{3 \sigma_{1}^{2}\left(\theta_{2}-\tau_{3}\right)^{2}}{64 \tau_{1} \tau_{2}}\right. \\
\left.\sigma_{2}=-\frac{\sigma_{1}\left(-30 \theta_{2} \sigma_{1} \tau_{3}+15 \theta_{2}^{2} \sigma_{1}+64 \theta_{1} \tau_{2}+\sigma_{1}\left(15 \tau_{3}^{2}+64 \tau_{1} \tau_{2}\right)\right)}{64 \tau_{2}}\right\} .
\end{gathered}
$$


We now using the Eq. (26) and Eqs. (15)-(19) in Eq. (20), and argue the following cases. If $\Omega<0$, then

$$
\begin{array}{r}
g_{1}(x, t)=\left\{\frac{3 \sigma_{1}\left(\theta_{2}-\tau_{3}\right)}{8 \tau_{2}} \mp \frac{3 i \sqrt{3} \sigma_{1}^{2}\left(\theta_{2}-\tau_{3}\right)^{2} \operatorname{coth}(\zeta \sqrt{-\Omega})}{128 \sqrt{\tau_{2} \tau_{1}} \tau_{2} \sqrt{-\Omega}} \pm \frac{i \sqrt{3} \sqrt{\tau_{1}} \sqrt{-\Omega} \tanh (\zeta \sqrt{-\Omega})}{2 \sqrt{\tau_{2}}}\right\}^{\frac{1}{2}} \\
\times \exp \left(\iota \frac{\Gamma(\beta+1)}{\mu}\left(-\sigma_{1} x^{\mu}+\sigma_{2} t^{\mu}\right)\right)
\end{array}
$$

or

$$
\begin{array}{r}
g_{2}(x, t)=\left\{\frac{3 \sigma_{1}\left(\theta_{2}-\tau_{3}\right)}{8 \tau_{2}} \mp \frac{3 i \sqrt{3} \sigma_{1}^{2}\left(\theta_{2}-\tau_{3}\right)^{2} \tanh (\zeta \sqrt{-\Omega})}{128 \sqrt{\tau_{2} \tau_{1}} \tau_{2} \sqrt{-\Omega}} \pm \frac{i \sqrt{3} \sqrt{\tau_{1}} \sqrt{-\Omega} \operatorname{coth}(\zeta \sqrt{-\Omega})}{2 \sqrt{\tau_{2}}}\right\}^{\frac{1}{2}} \\
\times \exp \left(\iota \frac{\Gamma(\beta+1)}{\mu}\left(-\sigma_{1} x^{\mu}+\sigma_{2} t^{\mu}\right)\right)
\end{array}
$$

If $\Omega>0$,then

$$
\begin{aligned}
g_{3}(x, t)=\left\{\frac{3 \sigma_{1}\left(\theta_{2}-\tau_{3}\right)}{8 \tau_{2}} \mp \frac{i \sqrt{3} \sqrt{\tau_{1}} \sqrt{\Omega} \tan (\zeta \sqrt{\Omega})}{2 \sqrt{\tau_{2}}}\right. & \left. \pm \frac{\cot (\zeta \sqrt{\Omega}) 3 i \sqrt{3} \sigma_{1}^{2}\left(\theta_{2}-\tau_{3}\right)^{2}}{128 \sqrt{\tau_{1} \tau_{2}} \tau_{2} \sqrt{\Omega}}\right\}^{\frac{1}{2}} \\
& \times \exp \left(\iota \frac{\Gamma(\beta+1)}{\mu}\left(-\sigma_{1} x^{\mu}+\sigma_{2} t^{\mu}\right)\right)
\end{aligned}
$$

or

$$
\begin{array}{r}
g_{4}(x, t)=\left\{\frac{3 \sigma_{1}\left(\theta_{2}-\tau_{3}\right)}{8 \tau_{2}} \pm \frac{\left(i \sqrt{3} \sqrt{\tau_{1}}\right) \sqrt{\Omega} \cot (\zeta \sqrt{\Omega})}{2 \sqrt{\tau_{2}}} \mp \frac{\tan (\zeta \sqrt{\Omega})\left(3 i \sqrt{3} \sigma_{1}^{2}\left(\theta_{2}-\tau_{3}\right)^{2}\right)}{128 \sqrt{\tau_{1} \tau_{2}} \tau_{2} \sqrt{\Omega}}\right\}^{\frac{1}{2}} \\
\times \exp \left(\iota \frac{\Gamma(\beta+1)}{\mu}\left(-\sigma_{1} x^{\mu}+\sigma_{2} t^{\mu}\right)\right) \quad(30)
\end{array}
$$

Set 3:

$$
\begin{aligned}
\left\{\alpha_{0}=\frac{3 \sigma_{1}\left(\theta_{2}-\tau_{3}\right)}{8 \tau_{2}}, \alpha_{1}\right. & =\mp \frac{i \sqrt{3} \sqrt{\tau_{1}}}{2 \sqrt{\tau_{2}}}, \beta_{1}= \pm \frac{3 i \sqrt{3} \sigma_{1}^{2}\left(\theta_{2}-\tau_{3}\right)^{2}}{128 \sqrt{\tau_{1}} \tau_{2}^{3 / 2}}, \Omega=\frac{3 \sigma_{1}^{2}\left(\theta_{2}-\tau_{3}\right)^{2}}{64 \tau_{1} \tau_{2}} \\
\sigma_{2} & \left.=-\frac{\sigma_{1}\left(-6 \theta_{2} \sigma_{1} \tau_{3}+3 \theta_{2}^{2} \sigma_{1}+16 \theta_{1} \tau_{2}+\sigma_{1}\left(3 \tau_{3}^{2}+16 \tau_{1} \tau_{2}\right)\right)}{16 \tau_{2}}\right\}
\end{aligned}
$$

We now using the Eq. (31) and Eqs. (15)-(19) in Eq. (20), and argue the following cases. If $\Omega<0$, then 


$$
\begin{array}{r}
g_{1}(x, t)=\left\{\frac{3 \sigma_{1}\left(\theta_{2}-\tau_{3}\right)}{8 \tau_{2}} \mp \frac{3 i \sqrt{3} \sigma_{1}^{2}\left(\theta_{2}-\tau_{3}\right)^{2} \operatorname{coth}(\zeta \sqrt{-\Omega})}{128 \sqrt{\tau_{2} \tau_{1}} \tau_{2} \sqrt{-\Omega}} \pm \frac{\left(i \sqrt{3} \sqrt{\tau_{1}} \sqrt{-\Omega}\right) \tanh (\zeta \sqrt{-\Omega})}{2 \sqrt{\tau_{2}}}\right\}^{\frac{1}{2}} \\
\times \exp \left(\iota \frac{\Gamma(\beta+1)}{\mu}\left(-\sigma_{1} x^{\mu}+\sigma_{2} t^{\mu}\right)\right)
\end{array}
$$

or

$$
\begin{array}{r}
g_{2}(x, t)=\left\{\frac{3 \sigma_{1}\left(\theta_{2}-\tau_{3}\right)}{8 \tau_{2}} \mp \frac{3 i \sqrt{3} \sigma_{1}^{2}\left(\theta_{2}-\tau_{3}\right)^{2} \tanh (\zeta \sqrt{-\Omega})}{128 \sqrt{\tau_{2} \tau_{1}} \tau_{2} \sqrt{-\Omega}} \pm \frac{\left(i \sqrt{3} \sqrt{\tau_{1}} \sqrt{-\Omega}\right) \operatorname{coth}(\zeta \sqrt{-\Omega})}{2 \sqrt{\tau_{2}}}\right\}^{\frac{1}{2}} \\
\times \exp \left(\iota \frac{\Gamma(\beta+1)}{\mu}\left(-\sigma_{1} x^{\mu}+\sigma_{2} t^{\mu}\right)\right)
\end{array}
$$

If $\Omega>0$,then

$$
\begin{array}{r}
g_{3}(x, t)=\left\{\frac{3 \sigma_{1}\left(\theta_{2}-\tau_{3}\right)}{8 \tau_{2}} \mp \frac{\left(i \sqrt{3} \sqrt{\tau_{1}}\right) \sqrt{\Omega} \tan (\zeta \sqrt{\Omega})}{2 \sqrt{\tau_{2}}} \pm \frac{\cot (\zeta \sqrt{\Omega})\left(3 i \sqrt{3} \sigma_{1}^{2}\left(\theta_{2}-\tau_{3}\right)^{2}\right)}{128 \sqrt{\tau_{1} \tau_{2}} \tau_{2} \sqrt{\Omega}}\right\}^{\frac{1}{2}} \\
\times \exp \left(\iota \frac{\Gamma(\beta+1)}{\mu}\left(-\sigma_{1} x^{\mu}+\sigma_{2} t^{\mu}\right)\right) \quad(34)
\end{array}
$$

or

$$
\begin{array}{r}
g_{4}(x, t)=\left\{\frac{3 \sigma_{1}\left(\theta_{2}-\tau_{3}\right)}{8 \tau_{2}} \pm \frac{\left(i \sqrt{3} \sqrt{\tau_{1}}\right) \sqrt{\Omega} \cot (\zeta \sqrt{\Omega})}{2 \sqrt{\tau_{2}}} \mp \frac{\tan (\zeta \sqrt{\Omega})\left(3 i \sqrt{3} \sigma_{1}^{2}\left(\theta_{2}-\tau_{3}\right)^{2}\right)}{128 \sqrt{\tau_{1} \tau_{2}} \tau_{2} \sqrt{\Omega}}\right\}^{\frac{1}{2}} \\
\times \exp \left(\iota \frac{\Gamma(\beta+1)}{\mu}\left(-\sigma_{1} x^{\mu}+\sigma_{2} t^{\mu}\right)\right) \quad(35)
\end{array}
$$

Set 4:

$$
\begin{aligned}
\left\{\alpha_{0}\right. & =\frac{3 \sigma_{1}\left(\theta_{2}-\tau_{3}\right)}{8 \tau_{2}}, \alpha_{1}=0, \beta_{1}=\mp \frac{3 i \sqrt{3} \sigma_{1}^{2}\left(\theta_{2}-\tau_{3}\right)^{2}}{32 \sqrt{\tau_{1}} \tau_{2}^{3 / 2}} \\
\sigma_{2} & \left.=-\frac{\sigma_{1}\left(-6 \theta_{2} \sigma_{1} \tau_{3}+3 \theta_{2}^{2} \sigma_{1}+16 \theta_{1} \tau_{2}+\sigma_{1}\left(3 \tau_{3}^{2}+16 \tau_{1} \tau_{2}\right)\right)}{16 \tau_{2}}, \Omega=\frac{3 \sigma_{1}^{2}\left(\theta_{2}-\tau_{3}\right)^{2}}{16 \tau_{1} \tau_{2}}\right\}
\end{aligned}
$$

We now using the Eq. (36) and Eqs. (15)-(19) in Eq. (20), and argue the following cases. If $\Omega<0$, then

$g_{1}(x, t)=\left\{\frac{\left(3 \sigma_{1}\left(\theta_{2}-\tau_{3}\right)\right)\left(1 \pm \frac{i \sqrt{3} \sigma_{1}\left(\theta_{2}-\tau_{3}\right) \operatorname{coth}(\zeta \sqrt{-\Omega})}{4 \sqrt{\tau_{2} \tau_{1}} \sqrt{-\Omega}}\right)}{8 \tau_{2}}\right\}^{\frac{1}{2}} \times \exp \left(\iota \frac{\Gamma(\beta+1)}{\mu}\left(-\sigma_{1} x^{\mu}+\sigma_{2} t^{\mu}\right)\right)$ 
or

$g_{2}(x, t)=\left\{\frac{\left(3 \sigma_{1}\left(\theta_{2}-\tau_{3}\right)\right)\left(1 \pm \frac{i \sqrt{3} \sigma_{1}\left(\theta_{2}-\tau_{3}\right) \tanh (\zeta \sqrt{-\Omega})}{4 \sqrt{\tau_{2} \tau_{1}} \sqrt{-\Omega}}\right)}{8 \tau_{2}}\right\}^{\frac{1}{2}} \times \exp \left(\iota \frac{\Gamma(\beta+1)}{\mu}\left(-\sigma_{1} x^{\mu}+\sigma_{2} t^{\mu}\right)\right)$

If $\Omega>0$, then

$g_{3}(x, t)=\left\{\frac{\left(3 \sigma_{1}\left(\theta_{2}-\tau_{3}\right)\right)\left(1 \mp \frac{i \sqrt{3} \sigma_{1}\left(\theta_{2}-\tau_{3}\right) \cot (\zeta \sqrt{\Omega})}{4 \sqrt{\tau_{2} \tau_{1}} \sqrt{\Omega}}\right)}{8 \tau_{2}}\right\}^{\frac{1}{2}} \times \exp \left(\iota \frac{\Gamma(\beta+1)}{\mu}\left(-\sigma_{1} x^{\mu}+\sigma_{2} t^{\mu}\right)\right)$,

or

$g_{4}(x, t)=\left\{\frac{\left(3 \sigma_{1}\left(\theta_{2}-\tau_{3}\right)\right)\left(1 \pm \frac{i \sqrt{3} \sigma_{1}\left(\theta_{2}-\tau_{3}\right) \tan (\zeta \sqrt{\Omega})}{4 \sqrt{\tau_{2} \tau_{1}} \sqrt{\Omega}}\right)}{8 \tau_{2}}\right\}^{\frac{1}{2}} \times \exp \left(\iota \frac{\Gamma(\beta+1)}{\mu}\left(-\sigma_{1} x^{\mu}+\sigma_{2} t^{\mu}\right)\right)$.

\subsection{Application of $\operatorname{Exp}_{a}$ function approach}

Here, we recall the main points of the aforesaid approach and then its demonstration has been exercised for required solutions. let's assume we have a NPDE in the following form:

$$
G\left(u, u^{2} u_{t}, u_{x}, u_{t t}, u_{x x}, u_{x t}, \ldots\right)=0
$$

The above PDE given in the Eq.(41) may be obtained in the below form of NODE :

$$
\Lambda\left(U, U^{\prime}, U^{\prime \prime}, \ldots,\right)=0,
$$

by implementing the below wave transformations:

$$
u(x, t)=U(\tau), \tau=b x+r t,
$$

Let us suppose a solution of Eq. (12) is of the below form $[9,10,12]$ :

$$
U(\tau)=\frac{\alpha_{0}+\alpha_{1} d^{\tau}+\ldots+\alpha_{m} d^{m \tau}}{\beta_{0}+\beta_{1} d^{\tau}+\ldots+\beta_{m} d^{m \tau}}, \quad d \neq 0,1
$$

here $\alpha_{i}$ and $\beta_{i}(0 \leq i \leq m)$ are unknown constants and to be find later. Positive integer $m$ is obtained by using the homogenous balance technique and balancing the highest derivative and nonlinear term in the Eq. (12). Putting Eq. (44) into non-linear Eq. (12), yields

$$
\wp\left(d^{\tau}\right)=\ell_{0}+\ell_{1} d^{\tau}+\ldots+\ell_{t} d^{t \tau}=0 .
$$


Putting $\ell_{i}(0 \leq i \leq t)$ in Eq. (45) equal to zero, a set of algebraic equations is gained as follows.

$$
\ell_{i}=0, \quad \text { where } i=0, \ldots, t .
$$

By this obtained sets, we get the nontrivial solutions of the NPDE (41).

For $m=1$, Eq. (44) reduces to:

$$
Q(\zeta)=\frac{\alpha_{0}+\alpha_{1} d^{\zeta}}{\beta_{0}+\beta_{1} d^{\zeta}}
$$

where $\alpha_{0}, \alpha_{1}, \beta_{0}$ and $\beta_{1}$ are unknowns. By putting Eq. (47) in the Eq. (5) and collecting the coefficients of each power of $d^{\zeta}$, yields the set of equations having $\alpha_{0}, \alpha_{1}, \beta_{1}$ and $\beta_{1}$ and other parameters. By using the soft computations, we gain the following sets:

Set 1:

$$
\begin{gathered}
\left\{\alpha_{0}=\alpha_{0}, \alpha_{1}=0, \beta_{0}=-\frac{2 i \alpha_{0} \sqrt{\tau_{2}}}{\sqrt{3} \sqrt{\tau_{1}} \log (d)}, \beta_{1}=\beta_{1}, \sigma_{1}=\frac{2 i \sqrt{\tau_{1}} \sqrt{\tau_{2}} \log (d)}{\sqrt{3}\left(\theta_{2}-\tau_{3}\right)}\right. \\
\begin{array}{r}
\sigma_{2}=\frac{\sqrt{\tau_{1}} \log (d)}{12\left(\theta_{2}-\tau_{3}\right)^{2}}\left(3 \theta_{2}^{2} \sqrt{\tau_{1}} \log (d)+\theta_{2}\left(-6 \sqrt{\tau_{1}} \tau_{3} \log (d)-8 i \sqrt{3} \theta_{1} \sqrt{\tau_{2}}\right)+16 \tau_{2} \tau_{1}^{3 / 2} \log (d)\right. \\
\left.\left.+3 \sqrt{\tau_{1}} \tau_{3}^{2} \log (d)+8 i \sqrt{3} \theta_{1} \sqrt{\tau_{2}} \tau_{3}\right)\right\}
\end{array} \\
g(x, t)=\left\{\frac{\alpha_{0}}{\beta_{1} d^{\zeta}-\frac{2 i \alpha_{0} \sqrt{\tau_{2}}}{\sqrt{3} \sqrt{\tau_{1} \log (d)}}}\right\}^{\frac{1}{2}} \times \exp \left(\iota \frac{\Gamma(\beta+1)}{\mu}\left(-\sigma_{1} x^{\mu}+\sigma_{2} t^{\mu}\right)\right)
\end{gathered}
$$

\section{Set 2:}

$$
\begin{gathered}
\left\{\alpha_{0}=\alpha_{0}, \alpha_{1}=0, \beta_{0}=\frac{2 i \alpha_{0} \sqrt{\tau_{2}}}{\sqrt{3} \sqrt{\tau_{1}} \log (d)}, \beta_{1}=\beta_{1}, \sigma_{1}=-\frac{2 i \sqrt{\tau_{1}} \sqrt{\tau_{2}} \log (d)}{\sqrt{3}\left(\theta_{2}-\tau_{3}\right)}\right. \\
\begin{array}{r}
\sigma_{2}=\frac{\sqrt{\tau_{1}} \log (d)}{12\left(\theta_{2}-\tau_{3}\right)^{2}}\left(3 \theta_{2}^{2} \sqrt{\tau_{1}} \log (d)+\theta_{2}\left(-6 \sqrt{\tau_{1}} \tau_{3} \log (d)+8 i \sqrt{3} \theta_{1} \sqrt{\tau_{2}}\right)+16 \tau_{2} \tau_{1}^{3 / 2} \log (d)\right. \\
\left.\left.+3 \sqrt{\tau_{1}} \tau_{3}^{2} \log (d)-8 i \sqrt{3} \theta_{1} \sqrt{\tau_{2}} \tau_{3}\right)\right\}
\end{array} \\
g(x, t)=\left\{\frac{\alpha_{0}}{\beta_{1} d^{\zeta}+\frac{2 i \alpha_{0} \sqrt{\tau_{2}}}{\sqrt{3} \sqrt{\tau_{1}} \log (d)}}\right\}^{\frac{1}{2}} \times \exp \left(\iota \frac{\Gamma(\beta+1)}{\mu}\left(-\sigma_{1} x^{\mu}+\sigma_{2} t^{\mu}\right)\right) .
\end{gathered}
$$

\section{Set 3:}

$$
\begin{aligned}
& \left\{\alpha_{0}=0, \alpha_{1}=\alpha_{1}, \beta_{0}=\beta_{0}, \beta_{1}=-\frac{2 i \alpha_{1} \sqrt{\tau_{2}}}{\sqrt{3} \sqrt{\tau_{1}} \log (d)}, \sigma_{1}=\frac{2 i \sqrt{\tau_{1}} \sqrt{\tau_{2}} \log (d)}{\sqrt{3}\left(\theta_{2}-\tau_{3}\right)}\right. \\
& \begin{array}{r}
\sigma_{2}=\frac{\sqrt{\tau_{1}} \log (d)}{12\left(\theta_{2}-\tau_{3}\right)^{2}}\left(3 \theta_{2}^{2} \sqrt{\tau_{1}} \log (d)+\theta_{2}\left(-6 \sqrt{\tau_{1}} \tau_{3} \log (d)-8 i \sqrt{3} \theta_{1} \sqrt{\tau_{2}}\right)+16 \tau_{2} \tau_{1}^{3 / 2} \log (d)\right. \\
\left.\left.+3 \sqrt{\tau_{1}} \tau_{3}^{2} \log (d)+8 i \sqrt{3} \theta_{1} \sqrt{\tau_{2}} \tau_{3}\right)\right\}
\end{array}
\end{aligned}
$$




$$
g(x, t)=\left\{\frac{\alpha_{1} d^{\zeta}}{\beta_{0}-\frac{2 i \alpha_{1} \sqrt{\tau_{2}} d^{\zeta}}{\sqrt{3} \sqrt{\tau_{1}} \log (d)}}\right\}^{\frac{1}{2}} \times \exp \left(\iota \frac{\Gamma(\beta+1)}{\mu}\left(-\sigma_{1} x^{\mu}+\sigma_{2} t^{\mu}\right)\right) .
$$

Set 4:

$$
\begin{aligned}
& \left\{\alpha_{0}=0, \alpha_{1}=\alpha_{1}, \beta_{0}=\beta_{0}, \beta_{1}=\frac{2 i \alpha_{1} \sqrt{\tau_{2}}}{\sqrt{3} \sqrt{\tau_{1}} \log (d)}, \sigma_{1}=-\frac{2 i \sqrt{\tau_{1}} \sqrt{\tau_{2}} \log (d)}{\sqrt{3}\left(\theta_{2}-\tau_{3}\right)},\right. \\
& \sigma_{2}=\frac{\sqrt{\tau_{1}} \log (d)}{12\left(\theta_{2}-\tau_{3}\right)^{2}}\left(3 \theta_{2}^{2} \sqrt{\tau_{1}} \log (d)+\theta_{2}\left(-6 \sqrt{\tau_{1}} \tau_{3} \log (d)+8 i \sqrt{3} \theta_{1} \sqrt{\tau_{2}}\right)+16 \tau_{2} \tau_{1}^{3 / 2} \log (d)\right. \\
& \left.\left.+3 \sqrt{\tau_{1}} \tau_{3}^{2} \log (d)-8 i \sqrt{3} \theta_{1} \sqrt{\tau_{2}} \tau_{3}\right)\right\} . \\
& g(x, t)=\left\{\frac{\alpha_{1} d^{\zeta}}{\beta_{0}+\frac{2 i \alpha_{1} \sqrt{\tau_{2}} d^{\zeta}}{\sqrt{3} \sqrt{\tau_{1}} \log (d)}}\right\}^{\frac{1}{2}} \times \exp \left(\iota \frac{\Gamma(\beta+1)}{\mu}\left(-\sigma_{1} x^{\mu}+\sigma_{2} t^{\mu}\right)\right) .
\end{aligned}
$$

\section{Results and discussion with graphical representation}

In this portion, we commenced notable graphs in 2-Dimension and 3-Dimension to explain the results given above. All of our results are consistent with the results found in $[21,26]$. The graph of (22) using the modified extended tanh expansion approach at $\mu=1, \lambda=$ $0.5, \beta=1, \sigma_{1}=0.1, \theta_{1}=-0.1, \theta_{2}=2, \tau_{1}=-0.1, \tau_{2}=1, \tau_{3}=0.1$ is introduced in Fig. 1. We shown the graph of (22) using the modified extended tanh expansion approach at
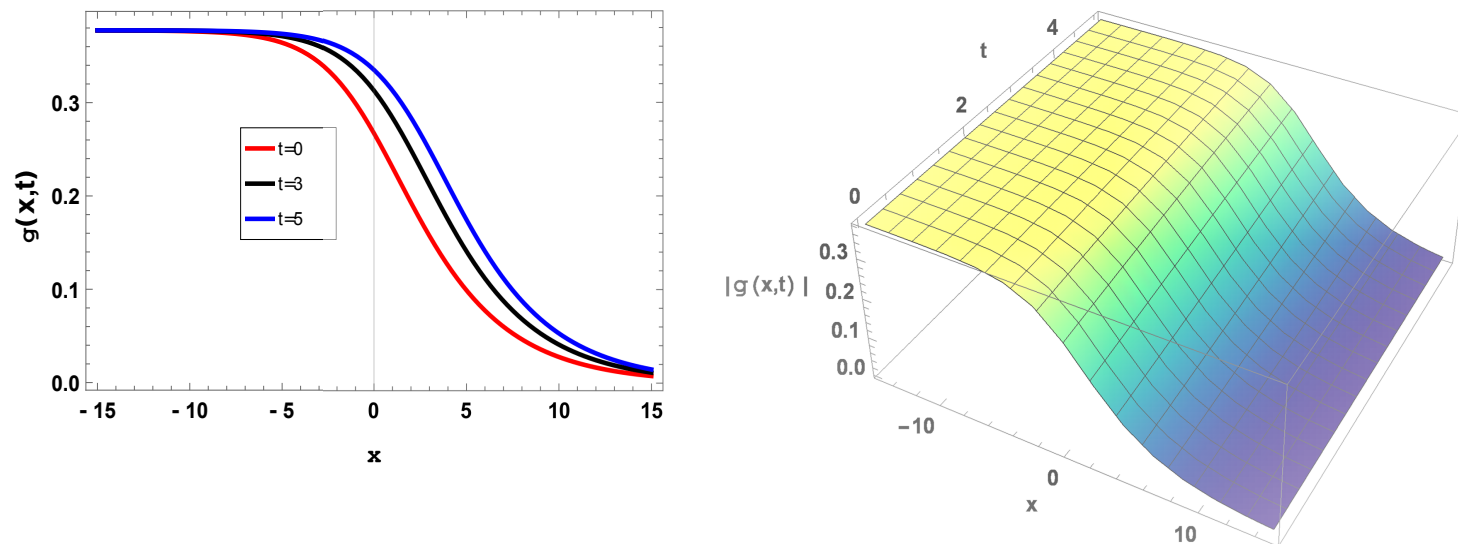

Figure 1: Graph of (22) using the modified extended tanh expansion approach at $\mu=$ $1, \lambda=0.5, \beta=1, \sigma_{1}=0.1, \theta_{1}=-0.1, \theta_{2}=2, \tau_{1}=-0.1, \tau_{2}=1, \tau_{3}=0.1$. 
$\lambda=0.5, \sigma_{1}=0.1, \theta_{1}=-0.1, \theta_{2}=2, \tau_{1}=-0.1, \tau_{2}=1, \tau_{3}=0.1$ with different values of $\mu$ and $\beta$ in Fig. 2. In Fig. 3 we show the graph of (37) using the modified extended tanh
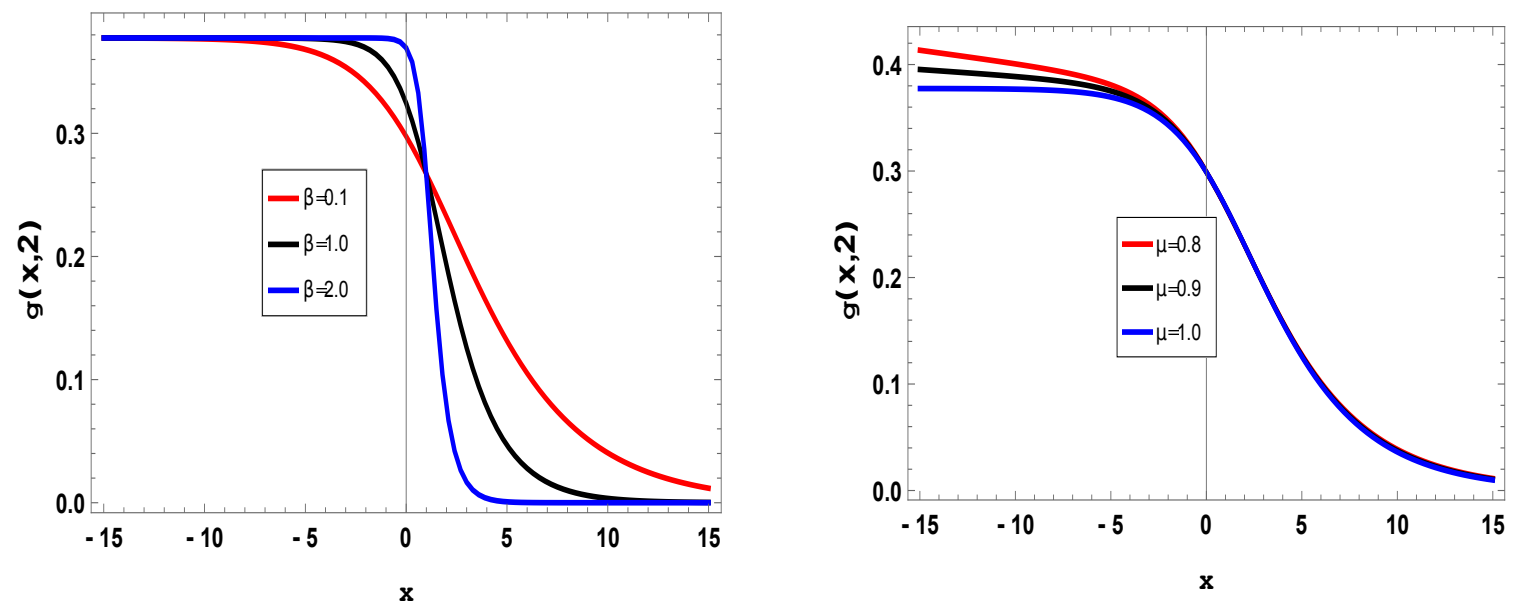

Figure 2: Graph of (22) using the modified extended tanh expansion approach at $\lambda=$ $0.5, \sigma_{1}=0.1, \theta_{1}=-0.1, \theta_{2}=2, \tau_{1}=-0.1, \tau_{2}=1, \tau_{3}=0.1$.

expansion approach at $\mu=1, \lambda=0.5, \beta=1, \sigma_{1}=0.1, \theta_{1}=-0.1, \theta_{2}=2, \tau_{1}=-0.1, \tau_{2}=$ $1, \tau_{3}=0.1$. Also, the graph of (37) using the modified extended tanh expansion approach
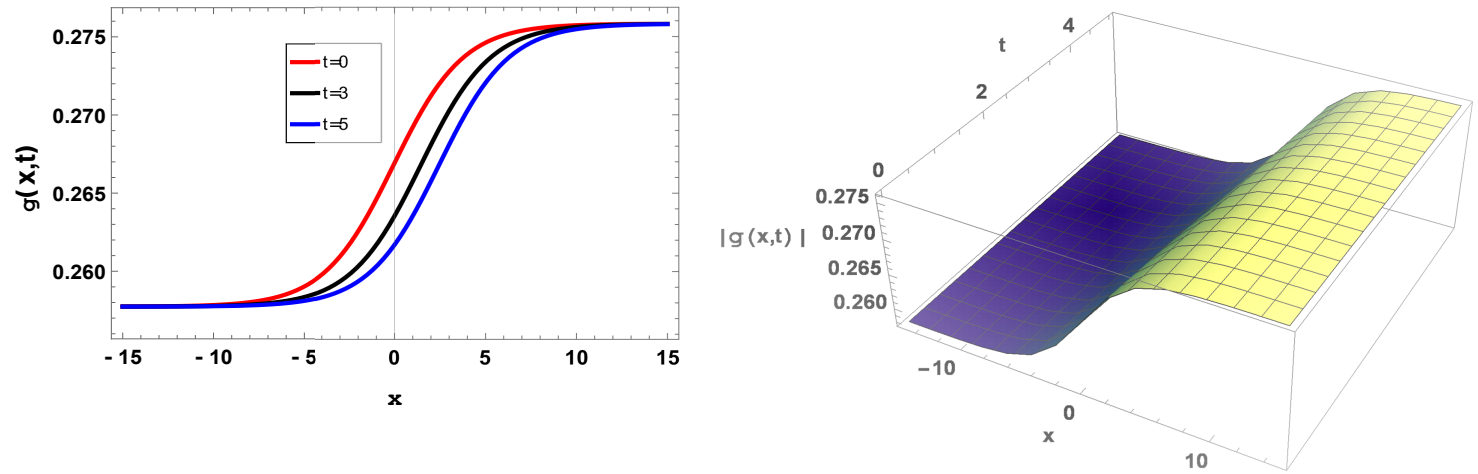

Figure 3: Graph of (37) using the modified extended tanh expansion approach at $\mu=$ $1, \lambda=0.5, \beta=1, \sigma_{1}=0.1, \theta_{1}=-0.1, \theta_{2}=2, \tau_{1}=-0.1, \tau_{2}=1, \tau_{3}=0.1$.

at $\lambda=0.5, \sigma_{1}=0.1, \theta_{1}=-0.1, \theta_{2}=2, \tau_{1}=-0.1, \tau_{2}=1, \tau_{3}=0.1$ is presented in Fig. 4 . The graph of (49) using the $\operatorname{Exp}_{a}$ function approach at $\lambda=0.5, \beta=1, \mu=1, \alpha_{0}=0.01$, $\theta_{1}=0.1, \theta_{2}=0.3, \tau_{1}=0.01, \tau_{2}=0.1, \tau_{3}=0.1, \beta_{1}=2, d=3$ is introduced in Fig. 5 , 

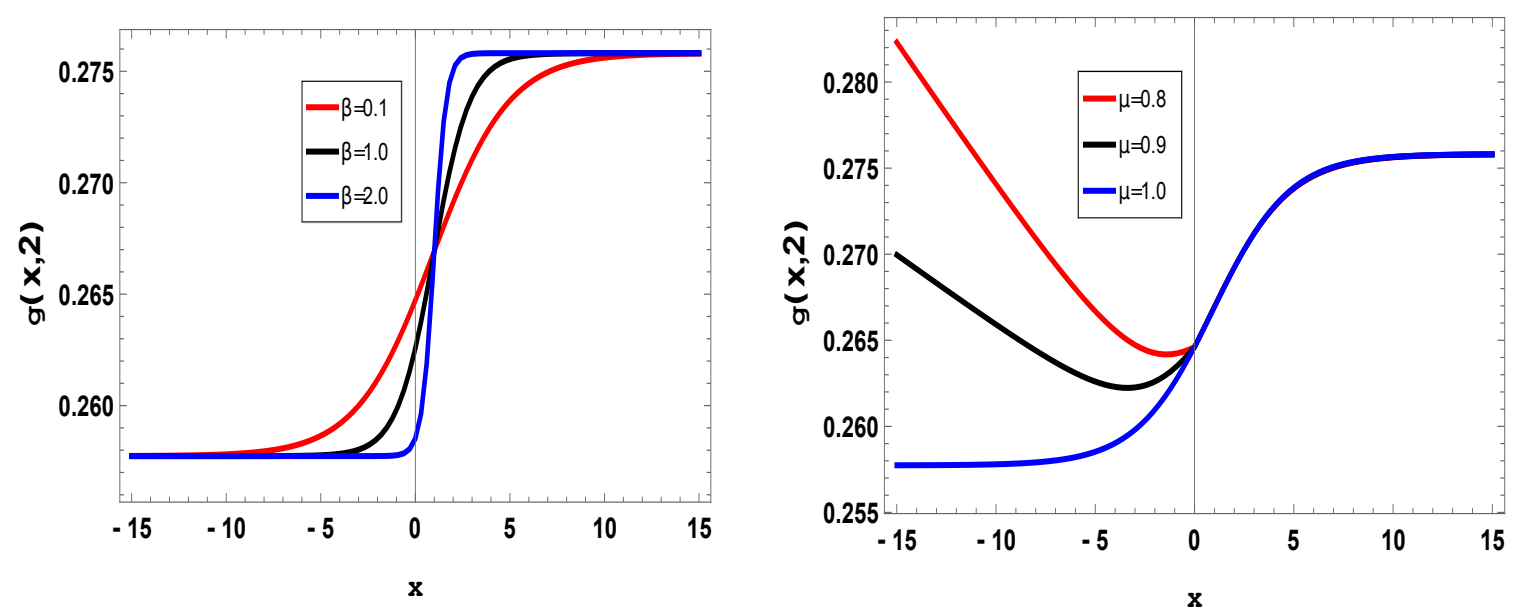

Figure 4: Graph of (37) using the modified extended tanh expansion approach at $\lambda=$ $0.5, \sigma_{1}=0.1, \theta_{1}=-0.1, \theta_{2}=2, \tau_{1}=-0.1, \tau_{2}=1, \tau_{3}=0.1$.
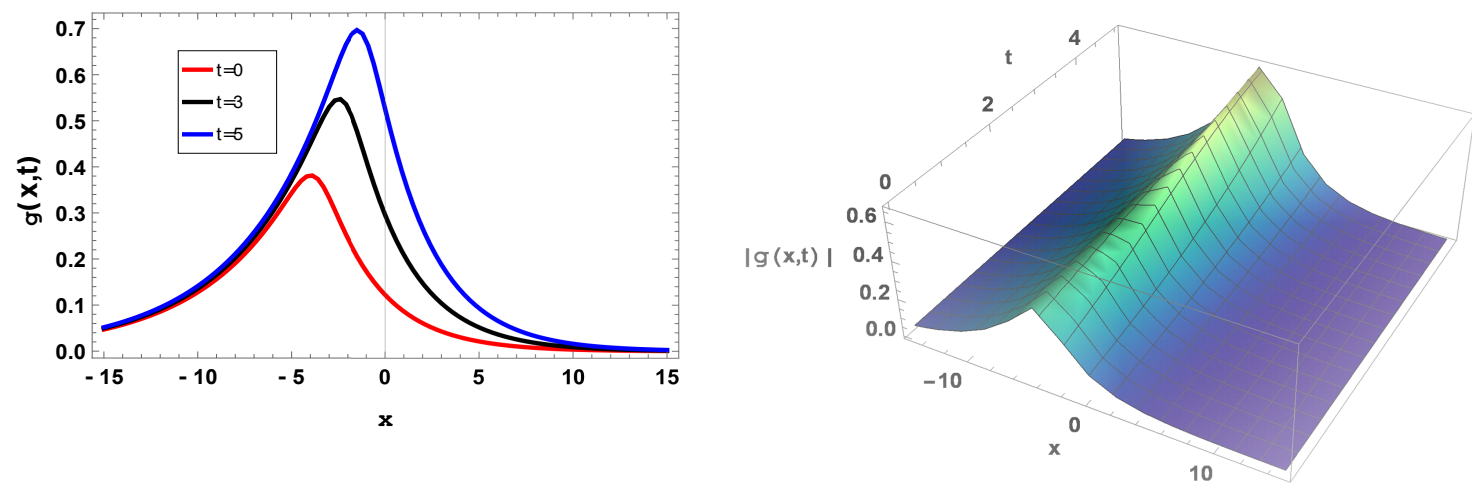

Figure 5: Graph of (49) using the $\operatorname{Exp}_{a}$ function approach at $\lambda=0.5, \beta=1, \mu=1, \alpha_{0}=$ $0.01, \theta_{1}=0.1, \theta_{2}=0.3, \tau_{1}=0.01, \tau_{2}=0.1, \tau_{3}=0.1, \beta_{1}=2, d=3$. 
We shown the graph of (49) using the $\operatorname{Exp}_{a}$ function approach at $\lambda=0.5, \alpha_{0}=0.01, \theta_{1}=$ $0.1, \theta_{2}=0.3, \tau_{1}=0.01, \tau_{2}=0.1, \tau_{3}=0.1, \beta_{1}=2, d=3$ with different values of $\mu$ and $\beta$ in Fig. 6 .
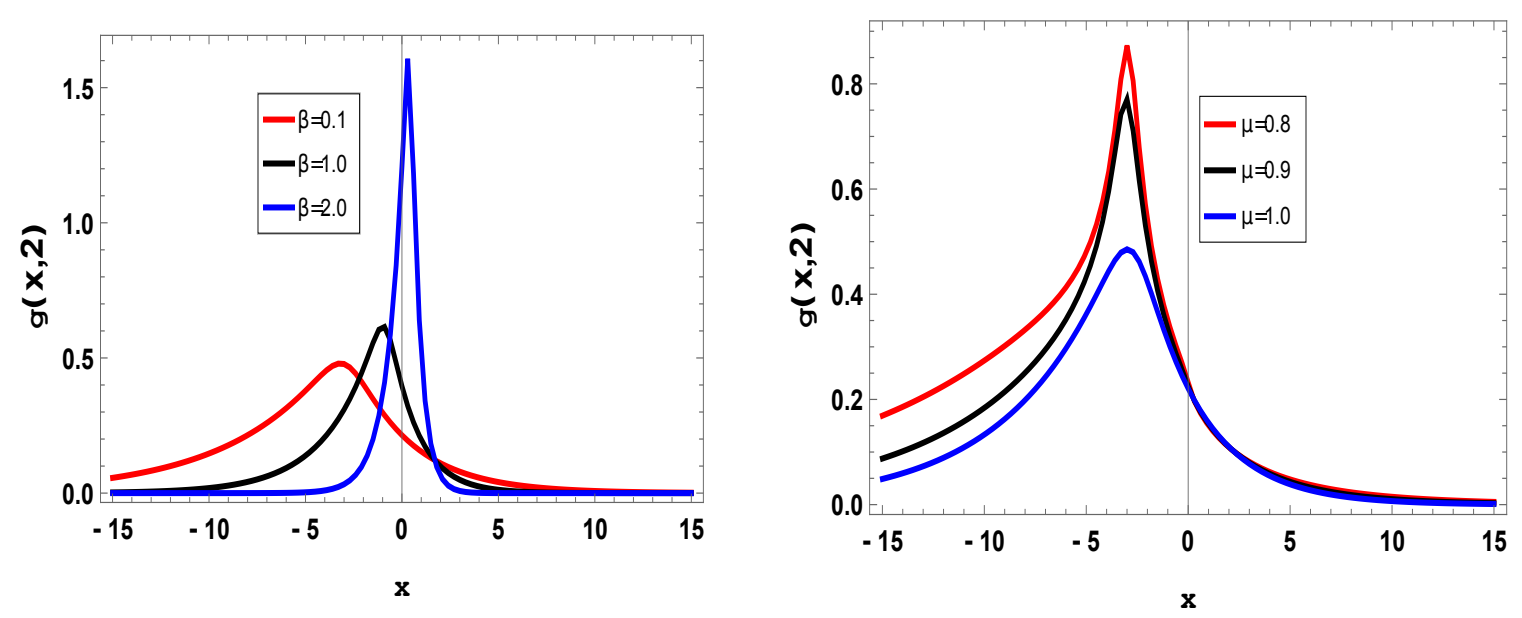

Figure 6: Graph of (49) using the $\operatorname{Exp}_{a}$ function approach at $\lambda=0.5, \alpha_{0}=0.01, \theta_{1}=$ $0.1, \theta_{2}=0.3, \tau_{1}=0.01, \tau_{2}=0.1, \tau_{3}=0.1, \beta_{1}=2, d=3$.

The graph of (55) using the $\operatorname{Exp}_{a}$ function approach at $\lambda=0.5, \beta=1, \mu=1, \alpha_{0}=$ 0.01, $\theta_{1}=0.1, \theta_{2}=0.3, \tau_{1}=0.01, \tau_{2}=0.1, \tau_{3}=0.1, \beta_{1}=2, d=3$ is introduced in Fig. 7 .
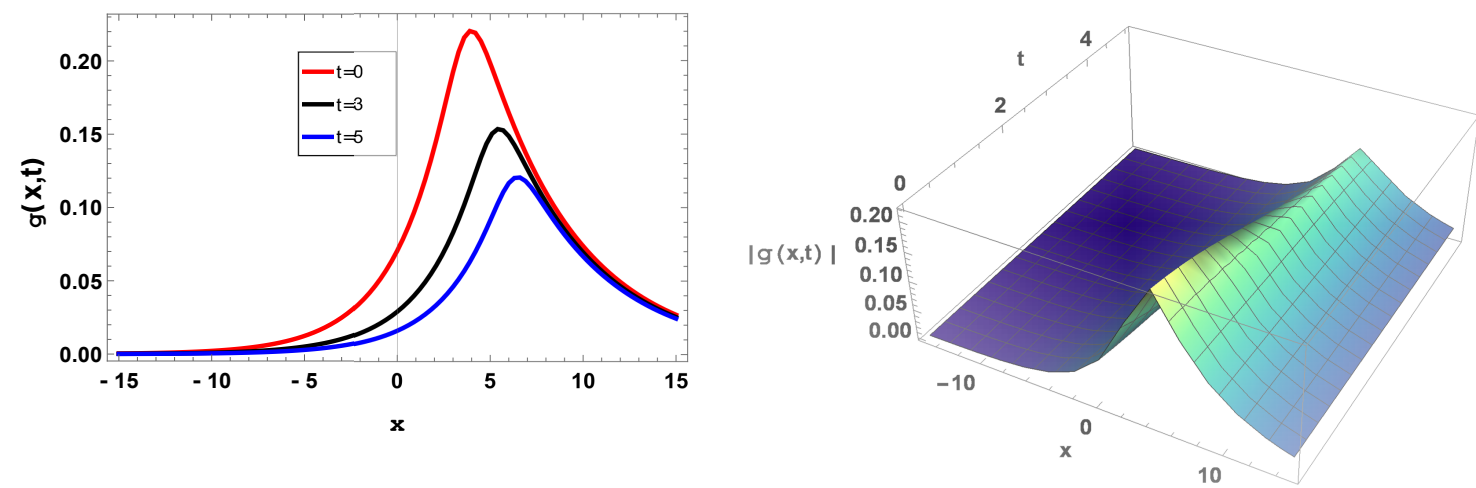

Figure 7: Graph of (55) using the $\operatorname{Exp}_{a}$ function approach at $\lambda=0.5, \beta=1, \mu=1, \alpha_{0}=$ $0.01, \theta_{1}=0.1, \theta_{2}=0.3, \tau_{1}=0.01, \tau_{2}=0.1, \tau_{3}=0.1, \beta_{1}=2, d=3$.

Finally, we introduced the graph of (55) using the $\operatorname{Exp}_{a}$ function approach at $\lambda=0.5, \alpha_{0}=$ $0.01, \theta_{1}=0.1, \theta_{2}=0.3, \tau_{1}=0.01, \tau_{2}=0.1, \tau_{3}=0.1, \beta_{1}=2, d=3$ with different values of 
$\mu$ and $\beta$ in Fig. 8.
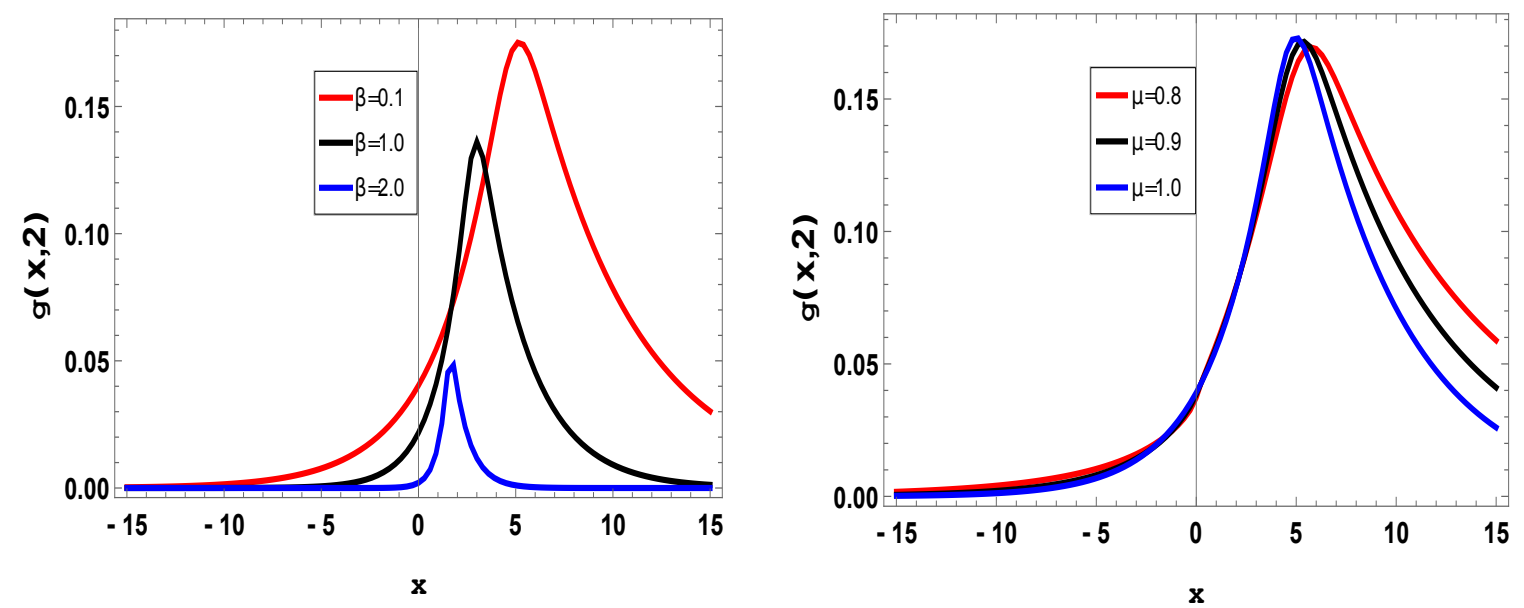

Figure 8: Graph of (55) using the $\operatorname{Exp}_{a}$ function approach at $\lambda=0.5, \alpha_{0}=0.01, \theta_{1}=$ $0.1, \theta_{2}=0.3, \tau_{1}=0.01, \tau_{2}=0.1, \tau_{3}=0.1, \beta_{1}=2, d=3$.

\section{Conclusion:}

In this paper, the perturbed Gerdjikov-Ivanov (GI) equation with conformable M-fractional derivative has been worked out and found its dark and other optical soliton solutions. For this, a fractional wave transformation was used for reducing the perturbed GI equation with truncated $M$-fractional derivative into a nonlinear ODE. Then, by applying modified extended tanh expansion and the $\operatorname{Exp}_{a}$ function methods, the novel soliton solutions are obtained. The achieved results are verified by software computation as well as explain with the help of 2-dimensional and 3-dimensional graphs.

\section{References}

[1] Asim Zafar, M. Raheel, Ahmed Bekir, Expolring the dark and singular soliton solutions of Biswas-Arshed model with full nonlinear form, Optik, 204, 164133 (2020).

[2] M.M Latha, V.C. Christal, An integrable model of $(2+1)$-dimensional Heisenberg ferromagnetic spin chain and soliton excitations, Phys.Scr., 89, 065204 (2014).

[3] K.R. Raslan, K. A. Khalid and M. A. Shallal, The modified extended tanh method with the Riccati equation for solving the space-time fractional EW and MEW equations, Chaos, Solitons and Fractals, 103, 404-409 (2017). 
[4] A. Bekir, Application of the extended tanh method for coupled nonlinear evolution equation, Commun. Nonlinear Sci., 13, 1742-1751, (2008).

[5] AM. Wazwaz, The tanh method for travelling wave solution of nonlinear equation, Appl. Math. comput., 154, 713-723, (2004).

[6] AM. Wazwaz, The extended tanh method for the Zakharov-Kuznestsov(ZK) equation, the modified ZK equation, and its generalized forms, Commun. Nonlinear Sci., 13, 1039-1047, (2008).

[7] E. Fan, Extended tanh-function method and its application to nonlinear equation, Phys. Lett. A., 277, 212-218 (2000).

[8] Fan, Engui and Hon, YC, Applications of extended tanh method to 'special' types of nonlinear equations, Applied Mathematics and Computation, 141, 2-3, 351-358 (2003).

[9] A. T. Ali and E.R. Hassan, General Expa-function method for nonlinear evolution equations, Applied Mathematics and Computation, 217(2) 451-459 (2010).

[10] E.M.E. Zayed and A.G. Al-Nowehy, Generalized kudryashov method and general $\exp _{a}$ function method for solving a high order nonlinear schrödinger equation, J. Space Explor, 6, 1-26 (2017).

[11] Hosseini, K. and Zabihi, A. and Samadani, F. and Ansari, R. New explicit exact solutions of the unstable nonlinear Schrödinger's equation using the $\exp _{a}$ and hyperbolic function methods, Optical and Quantum Electronics, 50, 2, 82 (2018).

[12] A. Zafar, The $\exp _{a}$ function method and the conformable time-fractional KdV equations, Nonlinear Engineering, 8, 728-732 (2019).

[13] Asim Zafar, Ahmed Bekir, Muhammad Raheel, Hadi Rezazadeh, Investigation for optical soliton solutions of two nonlinear Schrödinger equations via two concrete finite series methods, International Journal of Applied Computational Mathematics, 6, 3, $1-13(2020)$.

[14] Mainardi, Francesco and Gorenflo, Rudolf, On Mittag-Leffler-type functions in fractional evolution processes, Journal of Computational and Applied Mathematics, 118, 1-2, 283-299 (2000).

[15] Arshed S, Biswas A, Abdelaty M, Zhou Q, Moshokoa SP, Belic M.; Optical soliton perturbation for Gerdjikov-Ivanov equation via two analytical techniques, Chin J Phys., $56,2879-86$ (2018).

[16] Biswas A, Alqahtani RT., Chirp-free bright optical solitons for perturbed GerdjikovIvanov equation by semi-inverse variational principle, Optik 147 (2017). 
[17] Yasar E, Yıldırım Y, Yasar E., New optical solitons of space-time conformable fractional perturbed Gerdjikov-Ivanov equation by sine-Gordon equation method, Results Phys., 9, 1666-72 (2018).

[18] Biswas A, Ekici M, Sonmezoglu A, Majid FB, Triki H, Zhou Q, et al. Optical soliton perturbation for Gerdjikov-Ivanov equation by extended trial equation method, Optik, $158,747-52(2018)$.

[19] Arshed S., Two reliable techniques for the soliton solutions of perturbed GerdjikovIvanov equation, Optik, 164, 93-9 (2018).

[20] Kaur L, Wazwaz AM. Optical solitons for perturbed Gerdjikov-Ivanov equation, Optik, $174,447-51$ (2018).

[21] Hosseini K, Mirzazadeh M, Ilie M, Radmehr S., Dynamics of optical solitons in the perturbed Gerdjikov-Ivanov equation, Optik, 206, 164350 (2020).

[22] Wazwaz AM. Partial Differential Equations and Solitary Waves Theory. Berlin; Heidelberg, Springer Berlin Heidelberg, (2009).

[23] Arshad M, Seadawy AR, Lu D., Modulation stability and dispersive optical soliton solutions of higher order nonlinear Schrödinger equation and its applications in monomode optical fibers, Superlatt. Microstruct, 113, 419-29 (2018).

[24] Hosseini K, Samadani F, Kumar D, Faridi M., New optical solitons of cubic-quartic nonlinear Schrödinger equation, Optik, 157, 1101 (2018).

[25] González-Gaxiola O, Franco P, Bernal-Jaquez R., Solution of the nonlinear Schrödinger equation with defocusing strength nonlinearities through the LaplaceAdomian decomposition method, Int J Appl Comput Math, 3, 3723-43 (2017).

[26] Behzad Ghanbari and Dumitru Baleanu, New Optical Solutions of the Fractional Gerdjikov-Ivanov Equation With Conformable Derivative, Front. Phys., 8, 167 (2020). 